\title{
Descripción de las tres técnicas quirúrgicas más utilizadas en el manejo del hematoma subdural crónico
}

\author{
Comparison of three surgical techniques in management of subdural chronic \\ hematoma
}

\author{
Carlos Alberto Velasco López ${ }^{1}$ \\ Augusto Muñoz Caicedo ${ }^{2^{*}}$ \\ Laura Yulieth Santamaría Castillo ${ }^{3}$ \\ Laura Catalina Ruiz Mazuera ${ }^{4}$ \\ Nathalie Valencia Amaya ${ }^{5}$
}

Recibido: 20 de diciembre de 2016

Revisado: 04 de abril de 2017

Aceptado: 22 de junio de 2017

\section{Resumen}

Objetivo: Describir las tres técnicas quirúrgicas más utilizadas en el manejo del Hematoma Subdural Crónico (HSDC). Metodología: Estudio descriptivo de corte transversal realizado en 63 pacientes con diagnóstico de HSDC intervenidos quirúrgicamente mediante las técnicas trepano-drill, craneotomía con trefina y Craneotomía convencional, desde enero de 2013 hasta febrero de 2016 en una Institución Hospitalaria de la Ciudad de Popayán. Los datos obtenidos fueron analizados mediante el software de estadística SPSS-19. Resultados: De las tres técnicas utilizadas, la Trepano-Drill fue la más común (84,1\%). Además, los pacientes intervenidos mediante esta técnica presentaron una menor cantidad de complicaciones intrahospitalarias (26,4\%), su estado neurológico fue normal en el 77,4\% y solo el 34\% requirió de Unidad de Cuidados Intensivos. Conclusión: No se encontró diferencia estadísticamente significativa entre las tres técnicas utilizadas. Sin embargo, el drenaje del hematoma subdural crónico utilizando la técnica quirúrgica trepano-drill, mostró mayores ventajas sobre las otras dos técnicas evaluadas: craneotomía con trefina y craneotomía convencional.

Palabras clave: Hematoma subdural crónico, neurocirugía, craneotomía

\begin{abstract}
Objective: We aim to compare the three surgical techniques most commonly used in the management of Chronic Subdural Hematoma (HSDC). Methodology: A cross- sectional descriptive study performed in 63 patients with diagnosis of CSDH and intervened surgically from January 2013 to February 2016. The results were analyzed by the statistical package SPSS-19. Results: The trepano-drill technique was the most used $(88,9 \%)$ with the left hemisphere more affected $(43.4 \%)$, also it had some lower post-surgical complications. The majority of patients obtained a Karnofsky score between 100 and 70 points. Conclusion: Draining of the chronic subdural hematoma using trepano-Drill technique showed more advantages over trephine and conventional craniotomy, which made it the best option in the management of this event.
\end{abstract}

Key words: Hematoma, Subdural, Chronic, Neurosurgery, Decompressive Craniectomy, Craniotomy.

\footnotetext{
${ }^{1}$ Neurocirujano, Felow en cirugía de columna vertebral, profesor titular de la Universidad del Cauca, Colombia.

${ }^{2}$ Fonoaudiólogo, magister en Salud Pública, profesor asociado de la Universidad del Cauca, Colombia.

3,4,5 Estudiantes XIII semestre de Medicina, Universidad del Cauca, Colombia.

*Correspondencia: Augusto Muñoz Caicedo. Correo electrónico: amunozc@unicauca.edu.co
} 


\section{Introducción}

El hematoma subdural crónico es definido como una colección hemática localizada entre la duramadre y la aracnoides intracraneana, usualmente englobada por una membrana neoformada ${ }^{(1)}$, es más común en hombres ${ }^{(2)}$, su principal causa es el trauma craneoencefálico $^{(3,4)}$ y la aparición de los síntomas puede ocurrir unas semanas después de la lesión traumática $^{(5)}$. En Colombia se ha estimado una prevalencia de hematoma subdural crónico de 1.7 personas por cada 100.000 habitantes por año ${ }^{(6)}$..

Por lo general, el tratamiento y manejo de este tipo de hematomas se hace mediante la práctica de una craneotomía para el drenaje ${ }^{(7)}$. Sin embargo, existen diferentes técnicas quirúrgicas empleadas para el drenaje del HSDC, entre ellas, la craneotomía abierta, orificios de trepano-drill y microtrepano, y la craneotomía por trefina. Estudios han demostrado que la evacuación brusca del contenido del hematoma se acompaña de fenómenos vasculares como hematomas intracerebrales que pueden conducir a un daño isquémico severo ${ }^{(8)}$ y pueden incluso presentar recurrencias después del tratamiento quirúrgico. Algunos autores como Chater consideran la craneotomía como un factor de riesgo para la recurrencia ${ }^{(6)}$. Sin embargo, otros investigadores han establecido los beneficios de la técnica de trepano-drill, en comparación con la craneotomía convencional, estimando en general una recurrencia del $2.7 \%$ a $30 \%{ }^{(6)}$.

La morbilidad producida por los hematomas subdurales crónicos es aproximadamente $16 \%$ y la mortalidad $6.5 \%$, siendo el estado neurológico en el momento del diagnóstico, el factor pronóstico más significativo. A nivel local no se conocen datos sobre las técnicas quirúrgicas utilizadas para el manejo de este evento, razón por la cual se hizo necesario realizar un estudio para describir las tres técnicas quirúrgicas más utilizadas en el manejo del Hematoma Subdural Crónico (HSDC) en una IPS de III Nivel de complejidad de la ciudad de Popayán.

\section{Metodología}

Se realizó un estudio descriptivo retrospectivo de corte transversal en 63 pacientes con diagnóstico de hematoma subdural crónico e intervenidos quirúrgicamente en las salas de cirugía de un hospital de III nivel, mediante las técnicas: trepano-drill, craneotomía convencional y craneotomía con trefina, durante enero de 2013 y febrero de 2016. Se incluyeron pacientes con diagnóstico de hematoma subdural crónico a quienes se les realizó drenaje quirúrgico en la Clínica La Estancia; se excluyeron aquellos pacientes menores de 15 años, pacientes con alteraciones neurológicas previas al evento y pacientes con historia clínica incompleta. Una vez establecida la población, se dividió en tres grupos según la intervención quirúrgica realizada y se recolectaron los resultados en un instrumento diseñado por el grupo investigador. Los datos fueron obtenidos de la historia clínica de cada paciente y de una encuesta aplicada por vía telefónica con información primaria (paciente) y secundaria (familiar). Se controlaron los datos mediante el método de doble digitación en la base de datos de Excel y se trasladaron al paquete estadístico SPSS-19 para ser analizados y tabulados. Para establecer si la distribución de las variables entre los tres grupos independientes es homogénea y se ajusta a una distribución esperada se utilizó la prueba de Ji cuadrado.

El proyecto de investigación fue aprobado por el Comité de Ética de la Clínica la Estancia y desarrollado teniendo en cuenta los cuatro principios de la Bioética: la autonomía, la no maleficencia, la beneficencia y la justicia.

\section{Resultados}

Con respecto a las características quirúrgicas de los sujetos descritas en la tabla 1, el 88,9\% (56/63) de los pacientes fueron intervenidos bajo anestesia general y el 11,1\% (7/63) bajo anestesia local. La técnica quirúrgica más usada fue trepano-drill en el $84,1 \%$ (53/63) de los sujetos, seguido de craneotomía convencional en el $12,7 \%(8 / 63)$ y, por último, craneotomía con trefina en el 3,2\% (2/63). Al 16,9\% (9/53) de los pacientes intervenidos mediante la técnica trepano-drill se les realizó un agujero y al 83,1\% (44/53) 2 agujeros.

Así mismo, al 100\% (63/63) de los pacientes intervenidos quirúrgicamente se les instauró un dren en el espacio subdural, con una duración promedio de 3,0 días (Ds $\pm 1,1)$. Sin embargo, en el 90,5\% (57/63) de los casos, su duración estuvo entre 1 a 4 días y en el $9,5 \%$ $(6 / 63)$ de 5 a 8 días. En cuanto a la re-intervención quirúrgica, el 11,1\% (7/63) de los casos fueron re intervenidos; de estos, el 57,1\% (4/7) mediante la técnica de craneotomía convencional, el 28,6\% (2/7) con la técnica de trepano-drill y el 14,3\% (1/7) mediante craneotomía con trefina. 
Tabla 1. Características quirúrgicas de la población objeto de estudio.

\begin{tabular}{|l|c|c|}
\hline VARIABLES & $\mathbf{n = 6 3}$ & $\mathbf{\%}$ \\
\hline Tipo de anestesia & & \\
\hline General & 76 & 88,9 \\
\hline Local & & 11,1 \\
\hline Técnica quirúrgica & 53 & 84,1 \\
\hline Trepano-drill & 2 & 3,2 \\
\hline Craneotomía-trefina & 8 & 12,7 \\
\hline Craneotomía convencional & & \\
\hline Agujeros de trepano & 9 & 16,9 \\
\hline 1 agujero & 44 & 83,1 \\
\hline 2 agujeros & & \\
\hline Lateralidad del trepano & 23 & 43,4 \\
\hline Izquierdo & 20 & 37,7 \\
\hline Derecho & 10 & 18,9 \\
\hline Bilateral & & \\
\hline Uso de dren & 63 & 100,0 \\
\hline Si & & \\
\hline Días del dren & $3,0 \pm 1,1$ & \\
\hline Media \pm Ds & 57 & 90,5 \\
\hline $1-4$ & 6 & 9,5 \\
\hline $5-8$ & & \\
\hline Re intervención quirúrgica & 7 & 11,1 \\
\hline Si & 56 & 88,9 \\
\hline No & & \\
\hline Técnica de re intervención & 2 & 57,1 \\
\hline Craneotomía convencional & 1 & 14,3 \\
\hline Trepano - Drill & & \\
\hline Craneotomía con trefina & & \\
\hline
\end{tabular}

Las características clínicas postquirúrgicas según el tipo de técnica quirúrgica utilizada están descritas en la tabla 2. Con respecto al estado de conciencia de los pacientes valorado con la escala de Glasgow después del acto quirúrgico, el estudio encontró un puntaje 13 en el $69,8 \%(37 / 53)$ de los pacientes que fueron intervenidos con la técnica trepano-drill y del $50 \%(1 / 2)-$ (4/8) en los que fueron intervenidos con la técnica de craneotomía con trefina y craneotomía convencional, respectivamente.

Por otro lado, se evidenció que fue necesario realizar la TAC postoperatoria al 49,1\% (26/53) de los pacientes intervenidos con trepano-drill, al $100 \%(2 / 2)$ con craneotomía con trefina y al 87,5\% (7/8) de los pacientes con craneotomía convencional. La presencia de neumoencéfalo en los pacientes con TAC post operatorio fue de $96,1 \%(25 / 26)$ en los pacientes intervenidos con la técnica trepano-drill, $100 \%(2 / 2)$ con la craneotomía con trefina y $57,2 \%(4 / 7)$ con la craneotomía convencional.
Así mismo, en los pacientes con TAC post quirúrgico se presentó desviación de la línea media en el $42,3 \%$ $(11 / 26)$ de la técnica trepano - drill, $50 \%$ (1/2) en craneotomía con trefina y $57,1 \%(4 / 7)$ en craneotomía convencional; en el caso de compresión de ventrículos el $19,2 \%(5 / 26)$ de los pacientes intervenidos con trepanodrill, $0,0 \%(0 / 2)$ con la técnica de craneotomía con trefina y $28,5 \%(3 / 7)$ con craneotomía convencional. Por otra parte, se presentó borramiento de surcos en el $5,6 \%(3 / 26)$ de los pacientes intervenidos con trepanodrill y $0 \%$ de los intervenidos con las otras dos técnicas.

En cuanto a las complicaciones intrahospitalarias, solo el $26,4 \%(14 / 53)$ de los pacientes intervenidos con la técnica trepano-drill presentó alguna complicación, mientras que el $100 \%(2 / 2)$ de los intervenidos mediante la técnica craneotomía con trefina y el 62,5\% (5/8) de los intervenidos con la técnica craneotomía convencional presentaron alguna complicación como convulsión, neumonía y crisis hipertensiva. 
Descripción de las tres técnicas quirúrgicas más utilizadas en el manejo del hematoma subdural crónico

Tabla 2. Características clínicas postquirúrgicas según el tipo de técnica quirúrgica utilizada.

\begin{tabular}{|c|c|c|c|c|}
\hline \multirow[t]{2}{*}{ VARIABLES POSTOPERATORIAS } & $\begin{array}{c}\text { Trepano - } \\
\text { drill } \\
\mathbf{n}=53 \\
\%\end{array}$ & $\begin{array}{c}\text { Craneotomía } \\
\text { con trefina } \\
\mathbf{n}=2 \\
\%\end{array}$ & $\begin{array}{c}\text { Craneotomía } \\
\text { convencional } \\
n=8 \\
\%\end{array}$ & \multirow[t]{2}{*}{$\mathbf{P}$} \\
\hline & $\%$ & $\%$ & $\%$ & \\
\hline \multicolumn{5}{|l|}{ Glasgow postquirúrgico } \\
\hline$\leq 8$ & 17,0 & 50,0 & 37,5 & $\mathrm{ND}^{a}$ \\
\hline $9-12$ & 13,2 & 0,0 & 12,5 & $\mathrm{ND}^{a}$ \\
\hline$\geq 13$ & 69,8 & 50,0 & 50,0 & $\mathrm{ND}^{a}$ \\
\hline \multicolumn{5}{|l|}{ TAC postquirúrgico } \\
\hline $\mathrm{Si}$ & 49,1 & 100,0 & 87,5 & $\mathrm{ND}^{a}$ \\
\hline No & 50,9 & 0,0 & 12,5 & $\mathrm{ND}^{a}$ \\
\hline \multicolumn{5}{|l|}{$\begin{array}{l}\text { Presencia de neumoencéfalo en } \\
\text { pacientes con TAC postquirúrgico }\end{array}$} \\
\hline $\mathrm{Si}$ & 96,1 & 100,0 & 57,2 & $\mathrm{ND}^{a}$ \\
\hline No & 3,9 & 0,0 & 42,8 & $\mathrm{ND}^{a}$ \\
\hline \multicolumn{5}{|l|}{$\begin{array}{l}\text { Persistencia efecto masa en pa- } \\
\text { cientes con TAC postquirúrgico }\end{array}$} \\
\hline \multicolumn{5}{|l|}{ Desviación de la línea media } \\
\hline $\mathrm{Si}$ & 42,3 & 50,0 & 57,1 & $0,150^{\circ}$ \\
\hline No & 57,7 & 50,0 & 42,9 & \\
\hline \multicolumn{5}{|l|}{ Compresión de ventrículos } \\
\hline $\mathrm{Si}$ & 19,2 & 0,0 & 28,5 & $\mathrm{ND}^{a}$ \\
\hline No & 80,8 & 100,0 & 71,5 & \\
\hline \multicolumn{5}{|l|}{ Borramiento de los surcos } \\
\hline $\mathrm{Si}$ & 5,6 & 0,0 & 0,0 & $\mathrm{ND}^{a}$ \\
\hline No & 94,4 & 100,0 & 100,0 & \\
\hline \multicolumn{5}{|l|}{ Complicaciones intrahospitalarias } \\
\hline Alguna complicación & 26,4 & 100,0 & 62,5 & $\mathrm{ND}^{a}$ \\
\hline Ninguna complicación & 73,6 & 0,0 & 37,5 & $\mathrm{ND}^{a}$ \\
\hline
\end{tabular}

${ }^{a} \mathrm{ND}=$ No Determinado. ${ }^{b}$ Prueba de Ji cuadrado

Las características que indican el curso de la evolución postquirúrgica de los pacientes intervenidos se describen en la tabla 3 . El $34 \%(18 / 53)$ de los pacientes intervenidos mediante la técnica trepano-drill fueron llevados a Unidad de Cuidado Intensivo (UCI), el 13.2\% (7/53) a Unidad de Cuidado Intermedio (UCIN) y el $52.8 \%(28 / 53)$ a sala general. Por el contrario, el 50\% $(1 / 2)$ de los pacientes intervenidos por craneotomía con trefina fueron hospitalizados en UCI y el $50 \%(1 / 2)$ en sala general. Igualmente, el $75 \%(6 / 8)$ de los pacientes que fueron operados con craneotomía convencional fueron hospitalizados en UCI y el 25\% (2/8) en sala general. Con respecto al tiempo de hospitalización, el $92,5 \%$ (49/53) de los pacientes intervenidos con técnica trepano-drill, el 100\% (2/2) de los intervenidos con la técnica craneotomía por trefina y el 87,5\% (7/8) de los intervenidos con craneotomía convencional necesitaron menos de 20 días de hospitalización.

En cuanto a la recurrencia del hematoma, el $90.6 \%$ $(48 / 53)$, el $50,0 \%(1 / 2)$ y el $87.5 \%(7 / 8)$ de los pa- cientes intervenidos con las técnicas trepano-drill, craneotomía con trefina y craneotomía convencional respectivamente, no tuvo recurrencia. Por otro lado, la recurrencia temprana se presentó con mayor frecuencia en el $50 \%(1 / 2)$ de los intervenidos mediante la técnica craneotomía con trefina, mientras que la recurrencia tardía fue nula $(0,0 \%)$ en los intervenidos mediante las técnicas craneotomía con trefina y craneotomía convencional.

Con respecto al estado neurológico al momento del alta hospitalaria, el 86,7\% (46/53) de los intervenidos con trepano-drill no presentaron ninguna alteración. Sin embargo, $13.3 \%$ (7/53) presentó paresia, alteración del lenguaje o alteración del estado mental. Por otro lado, el $50 \%$ (1/2) de los intervenidos con craneotomía con trefina presentó alteración del estado mental y el $37,5 \%(3 / 8)$ de los operados con craneotomía convencional presentó paresia, alteración del lenguaje y alteración del estado mental. 
Se encontró una incidencia de muerte intrahospitalaria en el $9.4 \%(5 / 53)$ de los intervenidos con trepano-drill y del $25 \%(2 / 8)$ con craneotomía convencional. La descompensación cardiaca se presentó como causa de mortalidad en el $80 \%(42 / 53)$ de los intervenidos con trepano-drill y en el 50\% (4/8) de los intervenidos con craneotomía convencional.

Por otra parte, de los pacientes contactados y oper- ados con las técnicas trepano-drill, craneotomía con trefina y craneotomía convencional, el 20.8\%, el $50 \%$ y el $25 \%$ respectivamente, son capaces de trabajar. Sin embargo, en los pacientes operados con trepano-drill el $20.8 \%$ es incapaz de trabajar, pero se vale por sí mismo y, el $5.7 \%$ es incapaz de valerse por sí mismo. En los pacientes intervenidos con craneotomía convencional, el $12.5 \%$ es incapaz de valerse por sí mismo.

Tabla 3. Evolución postquirúrgica de la población intervenida según el tipo de técnica quirúrgica.

\begin{tabular}{|l|c|c|c|c|}
\hline \multicolumn{1}{|c|}{ VARIABLES } & $\begin{array}{c}\text { Trepano } \\
\text { drill } \\
\mathbf{n = 5 3}\end{array}$ & $\begin{array}{c}\text { Craneotomía } \\
\text { con trefina } \\
\mathbf{n = 2}\end{array}$ & $\begin{array}{c}\text { Craneotomía } \\
\text { convencional } \\
\mathbf{n = 8}\end{array}$ & $\mathbf{P}$ \\
\cline { 2 - 5 } & $\mathbf{\%}$ & $\mathbf{\%}$ & $\mathbf{7}$ & \\
\hline $\begin{array}{l}\text { Servicio de hospitalización } \\
\text { postquirúrgico }\end{array}$ & & & & \\
\hline UCI & 34,0 & 50,0 & 75,0 & $\mathrm{ND}^{a}$ \\
\hline UCIN & 13,2 & 0,0 & 0,0 & $\mathrm{ND}^{a}$ \\
\hline Sala general & 52,8 & 50,0 & 25,0 & $\mathrm{ND}^{a}$ \\
\hline Tiempo de hospitalización & & & & \\
\hline$<20$ días & 92,5 & 100,0 & 87,5 & $\mathrm{ND}^{a}$ \\
\hline$>21$ días & 7,5 & 0,0 & 12,5 & $\mathrm{ND}^{a}$ \\
\hline Recurrencia del hematoma & & & & \\
\hline No & 90,6 & 50,0 & 87,5 & $\mathrm{ND}^{a}$ \\
\hline Temprana & 7,5 & 50,0 & 12,5 & $\mathrm{ND}^{a}$ \\
\hline Tardía & 1,9 & 0,0 & 0,0 & $\mathrm{ND}^{a}$ \\
\hline Estado neurológico al alta & & & & \\
\hline Normal & 86,7 & 50,0 & 62,5 & $\mathrm{ND}^{a}$ \\
\hline Paresia & 5,7 & 0,0 & 12,5 & $\mathrm{ND}^{a}$ \\
\hline Alteración del lenguaje & 3,8 & 0,0 & 12,5 & $\mathrm{ND}^{a}$ \\
\hline Alteración del estado mental & 3,8 & 50,0 & 12,5 & $\mathrm{ND}^{a}$ \\
\hline Muerte intrahospitalaria & & & & \\
\hline Si & 9,4 & 0,0 & 25,0 & $\mathrm{ND}^{a}$ \\
\hline No & 90,6 & 100,0 & 75,0 & $\mathrm{ND}^{a}$ \\
\hline Causa de muerte & 80,0 & 0,0 & 50,0 & $\mathrm{ND}^{a}$ \\
\hline Descompensación cardiaca & 20,0 & 0,0 & 50,0 & $\mathrm{ND}^{a}$ \\
\hline Otro & & & & $\mathrm{ND}^{a}$ \\
\hline $\begin{array}{l}\text { Funcionalidad Extrahospita- } \\
\text { laria: Karnofsky }\end{array}$ & 20,8 & 50,0 & 25,0 & \\
\hline Capaz de trabajar & 20,8 & 0,0 & 0,0 & $\mathrm{ND}^{a}$ \\
\hline $\begin{array}{l}\text { Incapaz de trabajar pero se vale } \\
\text { por sí mismo }\end{array}$ & 5,7 & 0,0 & 12,5 & \\
\hline Incapaz de valerse por sí mismo & 52,7 & 50,0 & 62,5 & \\
\hline $\begin{array}{l}\text { Pacientes no contactables extra } \\
\text { hospitalariamente }\end{array}$ & & & & \\
\hline
\end{tabular}

${ }^{a} \mathrm{ND}=$ No Determinado

\section{Discusión}

El $88.9 \%$ de los pacientes fueron intervenidos bajo anestesia general, datos muy similares a otros estudios $^{(9)}$. Sin embargo, estos datos difieren de los reportes de otras investigaciones, que describen que el $87 \%$ de los pacientes fueron sometidos a anestesia local, con el fin de evitar complicaciones ${ }^{(10)}$. Al respecto, 
es recomendable que el tipo de anestesia elegida debe ser aquella que reduzca riesgos y la de mejor elección individualizando cada paciente, aunque podría depender de la técnica de drenaje del hematoma que cada neurocirujano elija.

La técnica más usada fue la trepano-drill, y a la mayoría de estos se les realizó dos agujeros, siendo resultados similares a lo reportado en otros estudios. Al respecto, no se encontró evidencia de un mejor resultado en la aplicación de uno o dos agujeros ${ }^{[10]}$. Sin embargo, se encontró que algunos investigadores realizaron el procedimiento quirúrgico mediante la técnica de trepano-drill empleando uno o dos agujeros y la craneotomía se reservaron para aquellos pacientes con hematoma subdural recurrente o formación de membranas ${ }^{(11)}$. De acuerdo a algunos autores ${ }^{(7,9)}$ las aproximaciones quirúrgicas mínimamente invasivas como el trepano-drill o el twist-drill son consideradas las técnicas de elección inicial en la mayoría de los pacientes, tanto en niños como en adultos. La técnica del twist-drill no es empleada en el área de estudio, por lo tanto no pudo ser evaluada.

En cuanto al uso del dren, el $100 \%$ de la población recibió dren post-operatorio con un promedio de duración de 3 días, lo cual concuerda con otros estudios $^{(10,12)}$. Algunos investigadores reportan que los HSDC se resuelven con tratamiento quirúrgico a través de 1 agujero de trepano con drenaje continuo durante las 12 a 48 horas siguientes a la intervención quirúrgica(13). De manera similar, otras investigaciones han demostrado que el drenaje posterior a la evacuación quirúrgica del hematoma probablemente conlleva un mejor pronóstico ${ }^{(14)}$.

La reintervención quirúrgica se realizó solo en $11.1 \%$ de los pacientes y fueron realizados por la técnica de craneotomía convencional en su mayoría, concordante con lo encontrado en otros estudios ${ }^{(10)}$. Lo anterior, podría indicar que los cirujanos reservan el procedimiento más invasivo para la segunda intervención ${ }^{(10)}$.

Es importante mencionar que en la tabla 2 y 3 se describen los resultados de algunas variables mediante las tres técnicas quirúrgicas. Sin embargo, dado que la población que conforma cada grupo no es estadísticamente comparable, la variación en sus resultados no es estadísticamente significativa. En ese sentido, se encontró que con la técnica de trepano-drill, un mayor número de pacientes $(69,8 \%)$ salieron con Glasgow $\geq 13$ a diferencia de los pacientes intervenidos con las otras dos técnicas. Al respecto, en la literatura no se encontró referencias sobre el Glasgow postoperatorio en las diferentes técnicas quirúrgicas.

Así mismo, menos del 50\% de los pacientes intervenidos con la técnica trepano-drill no necesitaron la TAC post quirúrgica, mientras que más del $80 \%$ de los pacientes intervenidos con las otras dos técnicas, la necesitaron. Es importante mencionar que en la institución donde se realizó este estudio, no se considera de manejo rutinario la TAC post operatoria. Contrario a lo mencionado en otra investigación ${ }^{(10)}$ donde se describe la TAC post quirúrgico a todos los pacientes dentro de los primeros 5 días y evidencia de persistencia de efecto masa en solo el $50 \%$ de la población. Lo anterior puede ser explicado debido a que las colecciones residuales post tratamiento son comunes ${ }^{(14)}$ y por eso algunos investigadores ${ }^{(8)}$, recomiendan no tomar TAC post quirúrgica como control, a no ser que el paciente presente deterioro neurológico dado que la cavidad del hematoma puede ser visible en la TAC incluso de 2 a 6 meses después de evacuarlo.

La presencia de neumoencéfalo fue mayor en los pacientes intervenidos con la técnica craneotomía con trefina y trepano-drill. Otra publicación documentó la presencia de neumoencéfalo sin encontrar relación con recurrencia, pero reporta que en la literatura se considera al neumoencéfalo como un pronóstico de pobre expansión cerebral y por lo tanto mayor probabilidad de recurrencia ${ }^{(10)}$.

En cuanto a las complicaciones intrahospitalarias de la población de estudio, se encontró que la técnica quirúrgica con mayor número de pacientes sin ninguna complicación fue la técnica de trepano-drill (73.6\%). Sin embargo, en aquellos que las presentaron se encontró principalmente convulsión y neumonía. Al respecto, algunos estudios ${ }^{(10)}$ reportan empiema subdural, mientras que otros ${ }^{(14)}$ convulsiones, hemorragia intracerebral, falla en re expansión cerebral, neumoencéfalo a tensión y en el caso de no ser tratados, empiema subdural.

Con respecto al servicio de hospitalización postquirúrgico, se identificó que los pacientes intervenidos con craneotomía convencional fueron los que mayormente requirieron hospitalización en UCI (75\%), mientras que más del 50\% de los pacientes intervenidos con la técnica trepano-drill, requirieron hospitalización en sala general. Al respecto, no se encontraron datos en la literatura revisada sobre el servicio de hospitalización al que acudían los pacientes post drenaje.

En el tiempo de hospitalización, este estudio encontró que la mayoría de pacientes tuvo una hospitalización inferior a 20 días. Los datos anteriores son similares a los reportados en otros estudios que describen una media de 12.8 días $^{(11)}$, demostrando que los pacientes sometidos a este tipo de intervenciones se ven damnificados al interrumpir su rutina personal, familiar y social.

Para la recurrencia del hematoma, la técnica craneotomía con trefina fue la más común (50\%), siendo contrario a lo reportado en otros estudios que documentaron un $17 \%{ }^{(10)}$. Además se encontró asociación de este evento con la persistencia del efecto masa en el TAC post operatorio. Es importante mencionar que algunos investigadores describen que, a pesar de ser 
fácilmente tratable con cirugía, el hematoma subdural crónico tiene una alta tasa de recurrencia, entre el $5 \%$ y el $30 \%{ }^{(14)}$. También refieren que la craneotomía se asoció a mayores tasas de complicación si se realizaba como primera opción de manejo, pero fue superior en términos de manejo de recurrencia con respecto a procedimientos mínimamente invasivos. Igualmente, el uso de dren tras haber evacuado al hematoma se asoció a una disminución significativa en la tasa de recurrencia ${ }^{(14)}$.

En lo concerniente a la muerte intrahospitalaria se encontró un mayor número de muertes en pacientes sometidos a craneotomía convencional (25.0\%), comparado con la técnica de trepano-drill y la craneotomía con trefina, siendo la principal causa de muerte la descompensación cardiaca (paro cardiaco). $\mathrm{Al}$ respecto, algunos estudios no refieren mortalidad en los pacientes ${ }^{(1,13)}$, sin embargo, en otras publicaciones $^{(10,15)}$ se describe un $4 \%$ con descompensación aguda sistémica relacionada con su pobre estado general, resangrado subdural agudo, sangrado intraparenquimatoso y tromboembolismo pulmonar, como principal causa de muerte. A nivel nacional, la tasa de mortalidad registra entre $3.2 \%$ y $6.5 \%{ }^{(8)}$, sin embargo, aunque algunos investigadores consideran al HSDC como una enfermedad "benigna", refieren una mortalidad de $0.5 \%$ al $8 \%$, con algunos reportes que sobrepasan el $10 \%{ }^{(14)}$.

Los pacientes contactados telefónicamente refirieron en su mayoría, no presentar secuelas post quirúrgicas, sobre todo los intervenidos mediante la técnica trepano dril y craneotomía convencional. En la literatura revisada no se encontró ningún estudio que evaluara la secuela post quirúrgica.

Con respecto a la funcionalidad extra hospitalaria medida según la escala de Karnofsky, este estudio encontró que más del 20,8\% de los pacientes fueron capaces de trabajar después del drenaje del hematoma subdural. Los datos anteriores, son muy inferiores a los reportados en otra investigación, donde se midió la calidad de vida según la escala de Karnofsky, obteniendo un índice medio de 84,35 puntos, lo que corresponde a pacientes capaces de trabajar ${ }^{(15)}$.

\section{Conclusiones}

Pese a que no se encontró diferencia estadísticamente significativa entre los grupos de pacientes sometidos a las tres técnicas quirúrgicas, los resultados de esta investigación muestran que los efectos de la técnica trepano-drill en el manejo quirúrgico del hematoma subdural crónico, describen una mejor evolución clínica medida por la puntuación con la escala de coma de Glasgow post-operatorio, disminución en la necesidad de TAC post-operatorio, en complicaciones postquirúrgicas y recurrencia del hematoma, además menor mortalidad. Así mismo, la mayoría de los pacientes no tuvieron alteración del estado neurológico al momento del alta, ni presentaron secuelas. Igual- mente, la funcionalidad del paciente en el ámbito extra hospitalario fue muy buena, siendo la mayoría capaces de trabajar de manera independiente en las actividades cotidianas.

\section{Agradecimientos}

El grupo investigador agradece a la Universidad del Cauca, a la Clínica la Estancia SA, quienes facilitaron las herramientas para desarrollar el proyecto investigativo. Igualmente a los estudiantes de medicina Osmar David Torres Casanova, Diana Carolina Urbano Albán y Ahmed Abell Yasell Jaen quienes colaboraron en la recolección de la información.

\section{Referencias}

1. Jaramillo E. Hematomas subdurales crónicos unilaterales: presentación de 36 casos tratados quirúrgicamente. Influencia del control de la postura durante el post-operatorio en las recidivas de los hematomas. Rev Med. 2007; 29 (1): $16-24$.

2. Oh J, Shim J, Yoon S, Lee K. Influence of gender on occurrence of chronic subdural hematoma: is it an effect of cranial asymmetry? Korean Neurotraumatology Society. 2014; 10(2): 82-85. doi: $10.13004 /$ kjnt.2014.10.2.82

3. Secretaría de Salud de México. Diagnóstico y tratamiento del hematoma subdural crónico traumático en pacientes mayores de 18 años de edad. México; 2010.

4. Lacerda A, Estenoz J, Borroto R. Hematoma subdural crónico. Resultados quirúrgicos en 2 años de trabajo. Rev Cubana Cir. 1999; 38(1): 57-61.

5. Yuan L, Jun X, An-hua w, Yun-jie W. Burr- hole craniotomy treating chronic subdural hematoma: a report of 398 cases. Chin J Traumatol. 2010 Oct $1 ; 13(5): 265-9$.

6. Sagobal R, Moscote L. Patobiologia del hematoma subdural crónico. Rev Electron Biomed / Electron J Biomed 2008; 2:65-71.

7. Velasco C. Decisiones neuroquirúrgicas en trauma cráneo encefálico. 2da edición. Colombia. 2014.

8. Chater G, González D, Peña G, Diaz J, Jiménez E, Hakim F. Hematomas subdurales crónicos tratados en el hospital universitario fundación santa fe de Bogotá. Neurocien Colom 2010; 17(4): 65-81.

9. Schwarz F, Loos F, Dunisch P, Sakr Y, Alsafatli D, Kalff R, Ewald C. Risk factors for reoperation after initial trepination in crhonic subdural hematomas. Clin Neurolog Neurosurg. 2015 Nov;138:66-71. doi: 10.1016/j.clineuro.2015.08.002. 
10. Leroy HA, Aboukais R, Reyns N, Bourgeois, Labreuche J, Duhamel A, Lejeune JP. Predictors of functional outcomes and recurrence of chronic subdural hematomas. J Clin Neurosci. 2015 ;22(12):1895900. doi: 10.1016/j.jocn.2015.03.064.

11. Huang Y, Yang K, Lee T, Liao C. Bilateral chronic subdural hematoma: what is the clinical significance. Int J Surg. 2013;11(7):544-8. doi: 10.1016/j.ijsu.2013.05.007

12. Oh HJ, Lee K, Yoon SM, Shim JJ, Yun G, Bae HG. Post-operativE course and recurrence of chronic subdural hematoma. J Korean Neurosurg Soc. 2010; 48(6): 518-523. 2010. doi: 10.3340/jkns.2010.48.6.518
13. Esquivel Miranda M, Arías Quirós M, Cartín Brenes M, Suárez-Sánchez MJ, Salazar-Sánchez L. Hematoma subdural crónico: análisis de 95 casos. Med. leg. 2012; 29 (1) : 17-21.

14. Alvis H, Alcalá G, Rubiano A, Moscote L. Hematoma subdural crónico: fisiopatología y manejo quirúrgico. Artículo de Reseña. Boletín Médico científico de la Asociación Médica de Puerto Rico. 58-63.

15. García MA, Pulido-Rivas P, Pascual-Garvi JM, Sola RG. Hematomas subdurales crónicos: arquitectura interna del hematoma como predictor de recidiva. Rev Neurol. 2014; 59:294-300. doi: $10.33588 /$ rn.5907.2014262. 